\title{
Effects of dexmedetomidine on immune response in patients undergoing radical and reconstructive surgery for oral cancer
}

\author{
LILI HUANG $^{1,2^{*}}$, CHUANQI QIN ${ }^{2 *}$, LI WANG $^{2}$, TIEJUN ZHANG ${ }^{2}$ and JIANGUO LI \\ ${ }^{1}$ Department of Intensive Care Unit, Zhongnan Hospital of Wuhan University, Wuhan, Hubei 430071; \\ ${ }^{2}$ Department of Anesthesiology, School and Hospital of Stomatology, Wuhan University, Wuhan, Hubei 430079, P.R. China
}

Received October 4, 2019; Accepted November 20, 2020

DOI: $10.3892 / 01.2020 .12367$

\begin{abstract}
Oral cancer is one of the most common malignancies in the world. The present study aimed to investigate the effects of dexmedetomidine on immune response in patients undergoing radical and reconstructive surgery for oral cancer. Patients were randomly divided into the dexmedetomidine and control groups. Within $15 \mathrm{~min}$ before anesthesia induction, dexmedetomidine was infused with a $0.5 \mu \mathrm{g} \cdot \mathrm{kg}^{-1}$ loading dose followed by a maintenance dose of $0.4 \mu \mathrm{g} \cdot \mathrm{kg}^{-1} \cdot \mathrm{h}^{-1}$ to the end of operation in the dexmedetomidine group, whereas the same volume of saline was administered in the control group. Blood samples were obtained at five time-points: $30 \mathrm{~min}$ Before induction $\left(\mathrm{T}_{0}\right), 1 \mathrm{~h}$ after induction $\left(\mathrm{T}_{1}\right)$, end of the operation $\left(\mathrm{T}_{2}\right)$ and $24\left(\mathrm{~T}_{3}\right)$ and $48 \mathrm{~h}\left(\mathrm{~T}_{4}\right)$ after the operation. The $\mathrm{T}$ lymphocyte subsets (including $\mathrm{CD}^{+}, \mathrm{CD}^{+}$and $\mathrm{CD}^{+}$cells) and $\mathrm{CD}^{+} / \mathrm{CD}^{+}$ratio, $\mathrm{B}$ lymphocytes, dendritic cells and myeloid-derived suppressor cells (MDSCs) were analyzed by flow cytometry. All immunological indicators, except $\mathrm{CD}^{+}$cells, significantly decreased between the two groups at $\mathrm{T}_{1-3}$ compared with $\mathrm{T}_{0}(\mathrm{P}<0.05)$. The percentages of $\mathrm{CD}^{+}, \mathrm{CD}^{+}$, dendritic cells and the $\mathrm{CD} 4^{+} / \mathrm{CD}^{+}$ratios were significantly higher at $\mathrm{T}_{2-4}$ and the percentages of MDSCs were significantly lower at $\mathrm{T}_{2-4}$ in the dexmedetomidine group compared with the control group (all $\mathrm{P}<0.05$ ). These findings suggested that dexmedetomidine can attenuate immunosuppression in patients undergoing radical and reconstructive surgery for oral cancer.
\end{abstract}

\section{Introduction}

Oral cancer is one of the most common tumors with the 5 -year survival rate that has remained at $50 \%$ in the last few

Correspondence to: Dr Jianguo Li, Department of Intensive Care Unit, Zhongnan Hospital of Wuhan University, 169 Donghu Road, Wuchang, Wuhan, Hubei 430071, P.R. China

E-mail: drjianguoli@163.com

${ }^{*}$ Contributed equally

Key words: dexmedetomidine, immunity, oral neoplasms decades (1). Oral cancer is primarily caused by habits of betel quid chewing, smoking and alcohol consumption in Southeast Asia (1). Though it is typically regarded as a disease of the elderly, it has been reported that the numbers of young patients have been increasing worldwide in recent years (2). Radical resection and immediate reconstruction are the mainstays of treatment for oral cancer. However, the surgical stress response is considered to directly induce immunosuppression by activating the hypothalamus-pituitary-adrenal axis and sympathetic nervous system (3), and residual tumor cells after surgery are likely to metastasize due to decreased immunity (4). Meanwhile, some anesthetics have direct suppressive impacts on innate and adaptive immunity (5-8). Therefore, selection of suitable anesthetics is important during the perioperative period. Dexmedetomidine is an $\alpha 2$-adrenergic receptor agonist with analgesic, sedative, anxiolytic and anti-sympatholytic properties $(9,10)$. Several studies have indicated that dexmedetomidine can regulate perioperative immune response in radical surgeries of breast, colon and gastric cancer (11-13). Nevertheless, the influence of dexmedetomidine on immune response in patients with oral malignant tumors during operation remains unclear.

The present study aimed to observe the level of immune cells through flow cytometry and to evaluate the effects of dexmedetomidine on immune response in patients undergoing radical operation and immediate reconstruction with forearm flap for oral cancer.

\section{Materials and methods}

Patients and grouping. The study was carried out at the School and Hospital of Stomatology of Wuhan University and conducted accordance with the Declaration of Helsinki between September 2018 and January 2019. It was approved by The Ethics Committee of School and Hospital of Stomatology, Wuhan University (Wuhan, China; approval no. IRB-2018B23) and registered in the Chinese Clinical Trial Registry (ChiCTR-1800018367). Written informed consent was provided by all participants before the trial. Patients classified as American Society of Anesthesiologist (ASA) physical status I to II (14) and aged 30-70 years were enrolled. All patients were scheduled for radical operation and immediate reconstruction with forearm flap under general anesthesia. None had a history of endocrine, immune or circulatory 
system diseases. Other exclusion criteria included recent or concurrent chemotherapy and a requirement for perioperative blood transfusion or perioperative treatment with immunomodulatory agents. Patients who developed severe surgical complications including infection or secondary surgery, were also excluded from study. Patients were randomly allocated to two groups using a computer-generated randomization list, the procedure of details was following: Before the patients were allocated into group, random numbers were generated by computer. Patients who were enrolled in this study were allocated in the order of random numbers, the even numbers were divided into control group and the odd numbers were divided into experimental group (15).

Methods of anesthesia. All patients were premedicated with anintramuscular injection of $0.01 \mathrm{mg} \cdot \mathrm{kg}^{-1}$ atropine at $30 \mathrm{~min}$ before anesthesia. In the operating room, the right subclavian vein was cannulated for central venous pressure monitoring and the radial artery was cannulated for real-time blood pressure monitoring. The electrocardiography, blood oxygen saturation $\left(\mathrm{SaO}_{2}\right)$, end-tidal carbon dioxide $\left(\mathrm{P}_{\mathrm{et}} \mathrm{CO}_{2}\right)$, and cerebral state index were continuously monitored during the operation. Within $15 \mathrm{~min}$ before anesthesia induction, dexmedetomidine (lot no. 180604BP; Jiangsu Hengrui Medicine Co. Ltd.) was infused with a $0.5 \mu \mathrm{g} \cdot \mathrm{kg}^{-1}$ loading dose followed by a maintenance dose of $0.4 \mu \mathrm{g} \cdot \mathrm{kg}^{-1} \cdot \mathrm{h}^{-1}$ to the end of operation in the dexmedetomidine group. According to its clinical pharmacokinetics and pharmacodynamics (10), dexmedetomidine was actually discontinued $30 \mathrm{~min}$ before the surgery. But in the present study research, it was difficult to determine $30 \mathrm{~min}$ before the operation is completed. Therefore, in order to reduce deviation, the dexmedetomidine infusion was stopped immediately after the surgery with the opinion of making the baseline of every patient the same. The same volume of normal saline was administered to the patients in the control group. Anesthesia induction was performed by intravenous injection of propofol $1.5-2.0 \mathrm{mg} \cdot \mathrm{kg}^{-1}$, sufentanil $0.4 \mu \mathrm{g} \cdot \mathrm{kg}^{-1}$ and cisatracurium $0.2 \mathrm{mg} \cdot \mathrm{kg}^{-1}$ to facilitate nasal tracheal intubation. Anesthesia maintenance included sevoflurane $2-3 \%$, remifentanil $0.2-0.3 \mu \mathrm{g} \cdot \mathrm{kg}^{-1} \cdot \mathrm{min}^{-1}$ and intermittent injection of cisatracurium $0.1 \mathrm{mg} \cdot \mathrm{kg}^{-1}$. Mechanical ventilation was performed to maintain the $\mathrm{P}_{\mathrm{et}} \mathrm{CO}_{2}$ at $35-40 \mathrm{mmHg}$ and $\mathrm{SaO}_{2}>98 \%$. Blood pressure and heart rate were fluctuated within $\pm 120 \%$ of baseline values. The depth of anesthesia was monitored to maintain the cerebral state index between 40 and 60 . Sufentanil $0.1 \mu \mathrm{g} \cdot \mathrm{kg}^{-1}$ was administered as a loading dose for postoperative analgesia in each patient $30 \mathrm{~min}$ before the end of surgery, and patients received the same intravenous analgesia for postoperative pain therapy.

Peripheral blood mononuclear cell separation. Saphenous vein blood $(2 \mathrm{ml})$ was obtained at five time-points: $30 \mathrm{~min}$ before induction $\left(\mathrm{T}_{0}\right), 1 \mathrm{~h}$ after induction $\left(\mathrm{T}_{1}\right)$, end of the operation $\left(\mathrm{T}_{2}\right)$ and $24\left(\mathrm{~T}_{3}\right)$ and $48 \mathrm{~h}\left(\mathrm{~T}_{4}\right)$ after the operation. Peripheral blood mononuclear cells (PBMC) were isolated by centrifugation at $800 \mathrm{x}$ g for $20 \mathrm{~min}$ at room temperature using Lymphoprep $^{\mathrm{TM}}$ (Stemcell Technologies, Inc.).

Flow cytometry. The PBMCs were stained by the following antibodies at $4^{\circ} \mathrm{C}$ for $30 \mathrm{~min}$ : APC-eFluor 780-conjugated
anti-CD45 (clone: HI30; 1:100), Alexa Fluor 700-conjugated anti-CD3 (clone: UCHT1; 1:50), FITC-conjugated anti-CD4 (clone: RPA-T4; 1:200), PE-Cy7-conjugated anti-CD8 (clone: SK1; 1:200), PC5.5-conjugated anti-CD19 (clone: SJ25C1; 1:50), APC-conjugated anti-HLA-DR (clone: LN3; 1:50), PC5.5-conjugated anti-CD14 (clone: 61D3; 1:50), PE-conjugated anti-CD15 (clone: HI98; 1:50) and BV421-conjugated anti-CD11C (clone: 3.9; 1:50). PE-conjugated anti-CD15 were obtained from Becton, Dickinson and Company; BV421-conjugated anti-CD11C were obtained from BioLegend, Inc; APC-eFluor 780-conjugated anti-CD45, Alexa Fluor 700-conjugated anti-CD3, FITC-conjugated anti-CD4, PE-Cy7-conjugated anti-CD8, PC5.5-conjugated anti-CD19, PC5.5-conjugated anti-CD14 and APC-conjugated anti-HLA-DR were obtained from eBioscience; Thermo Fisher Scientific, Inc. Isotype-matched IgG controls (cat. no. 56-0038-41) were purchased from eBioscience; Thermo Fisher Scientific, Inc., and were incubated at $4^{\circ} \mathrm{C}$ for $30 \mathrm{~min}$. Dead cells were excluded using staining with Fixable Viability Dye eFluor ${ }^{\mathrm{TM}} 506$ at $4^{\circ} \mathrm{C}$ (eBioscience; Thermo Fisher Scientific, Inc.). Flow cytometry detection were performed on CytoFLEX flow cytometer (Beckman Coulter). The data were analyzed using FlowJo version 10 (Tree Star, Inc.) and gated by the side scatter and forward scatter filters.

Observational index. The levels of T lymphocyte subsets, B lymphocytes, dendritic cells and myeloid-derived suppressor cells (MDSCs) of the patients in the two groups were observed by flow cytometry. The level of agitation during emergence was assessed using the Richmond Agitation-Sedation Scale (RASS), which is defined as RASS score $\geq+2$ (16). The incidence of emergence agitation, hypotension and bradycardia during surgery were also recorded.

Statistical analysis. According to the percentages of $\mathrm{CD}^{+}$cells at $\mathrm{T}_{4}$ between two groups from our preliminary experiment, using Chinese High Intellectualized Statistical Software, with a type-I error of $5 \%$ and a power of $80 \%, 29$ patients were needed in each group. Anticipating a $10 \%$ dropout rate, a total of 70 patients were recruited. All statistical analyses were performed using GraphPad Prism 7 (GraphPad Software, Inc.). Data are expressed as mean \pm standard deviation or value. Differences in sex, ASA status and stage of tumor were compared using $\chi^{2}$ tests and Fisher's exact test as appropriate. Student's unpaired t-tests were performed to compare age, body mass index (BMI), duration of surgery, blood loss and liquid infusion volume. The differences in percentages of immune cells between time-points and the two groups are compared using two-way ANOVA and Bonferroni's correction. $\mathrm{P}<0.05$ was considered to indicate a statistically significant difference.

\section{Results}

Comparison of general data between two groups. A total of 70 patients were recruited, two refused to consent and two patients from each group were excluded due to blood transfusions. Therefore, 32 patients were left in each group (Fig. 1). The demographics and surgical profiles of the patients were 
Table I. Demographics and surgical profiles of the patients.

\begin{tabular}{lccc}
\hline Variables & Group D & Group C & P-value \\
\hline Age, years & $49 \pm 10$ & $51 \pm 11$ & 0.454 \\
Sex, male/female & $25 / 7$ & $24 / 8$ & 0.768 \\
BMI, kg/m ${ }^{2}$ & $22.9 \pm 3.5$ & $21.81 \pm 2.7$ & 0.159 \\
ASA status, I/II & $22 / 10$ & $23 / 9$ & 0.784 \\
Stage of tumor & & & 0.963 \\
$\mathrm{~T}_{1} \mathrm{~N}_{0} \mathrm{M}_{0}$ & 12 & 11 & \\
$\mathrm{~T}_{2} \mathrm{~N}_{0} \mathrm{M}_{0}$ & 15 & 16 & \\
$\mathrm{~T}_{3} \mathrm{~N}_{0} \mathrm{M}_{0}$ & 5 & 5 & \\
Duration of surgery, h & $6.6 \pm 0.5$ & $6.4 \pm 0.6$ & 0.108 \\
Blood loss, ml & $302 \pm 53$ & $280 \pm 54$ & 0.080 \\
Liquid infusion volume, $\mathrm{ml}$ & $3006 \pm 320$ & $3072 \pm 295$ & 0.336
\end{tabular}

BMI, Body Mass Index; ASA, American Society of Anesthesiologists; D, dexmedetomidine, C, control.

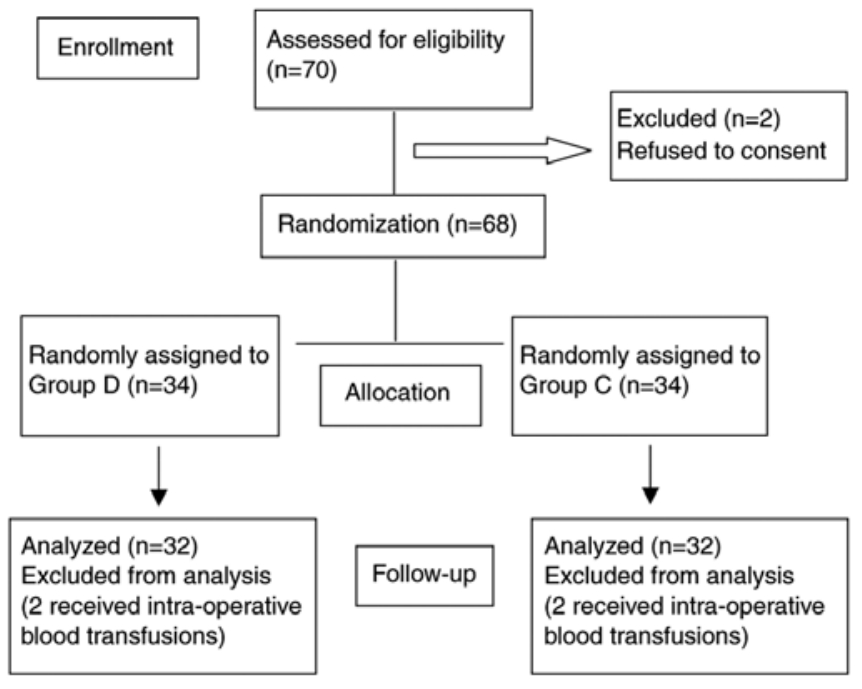

Figure 1. Consolidated Standards of Reporting Trials flow diagram of the patients included in the study. D, dexmedetomidine; $\mathrm{C}$, control.

similar in the two groups. The patients in the two groups showed no significant differences in age, sex, BMI, ASA, stage of tumor, duration of surgery, blood loss or liquid infusion volume (all $\mathrm{P}>0.05$; Table I).

Comparison of $T$ lymphocyte subsets between the two groups. No significant differences in the percentages of $\mathrm{CD}^{+}$and $\mathrm{CD}^{+}{ }^{+}$cells and the $\mathrm{CD} 4^{+} / \mathrm{CD}^{+}$ratios were observed between the two groups before anesthesia induction $(\mathrm{P}>0.05)$. The percentages of $\mathrm{CD}^{+}$and $\mathrm{CD}^{+}$cells, and the $\mathrm{CD}^{+} / \mathrm{CD}^{+}$ratios significantly decreased at $\mathrm{T}_{1-4}$ in the two groups compared with the baseline value at $T_{0}$ and significantly increased at $\mathrm{T}_{1-4}$ in the dexmedetomidine group compared with the control group (all $\mathrm{P}<0.05$ ). No significant difference in the percentage of $\mathrm{CD}^{+}$cells was found between the two groups at $\mathrm{T}_{0-4}(\mathrm{P}>0.05)$ (Fig. 2 and Fig. S1). These results indicated that cellular immunity was suppressed in the two groups after anesthesia and dexmedetomidine may be associated with less impairment of cellular immunity in these patients.

Comparison of $B$ lymphocytes between the two groups. The percentages of $B$ lymphocytes at $\mathrm{T}_{1-4}$ were significantly lower compared with those at $\mathrm{T}_{0}$ in the two groups (all $\mathrm{P}<0.05$ ), but no statistically significant differences were found between the two groups at the same time-points (P>0.05) (Fig. 3). These results implied that dexmedetomidine exerts minimal effect on the humoral immune response of patients undergoing radical and reconstructive surgery for oral cancer.

Comparison of dendritic cells between the two groups. The percentages of dendritic cells were significantly decreased at $\mathrm{T}_{1-4}$ in the two groups compared with the baseline value at $\mathrm{T}_{0}$, and they were significantly higher at $\mathrm{T}_{2-4}$ in the dexmedetomidine group compared with the respective control group $\left(0.5 \pm 0.2 \%\right.$ vs. $0.3 \pm 0.2 \%$ at $\mathrm{T}_{2}, 0.5 \pm 0.1 \%$ vs. $0.4 \pm 0.2 \%$ at $\mathrm{T}_{3}, 0.6 \pm 0.2 \%$ vs. $0.5 \pm 0.3 \%$ at $\mathrm{T}_{4}$; all $\mathrm{P}<0.05$; Fig. 4 ). These results suggested that dexmedetomidine may attenuate the inhibition of immune response and may be beneficial to antitumor therapy.

Comparison of MDSCs between the two groups. The percentages of MDSCs significantly decreased at $\mathrm{T}_{1-3}$ in the two groups compared with the respective baseline value at $\mathrm{T}_{0}$, and they were significantly lower at $\mathrm{T}_{2-4}$ in the dexmedetomidine group compared with the respective control group $\left(2.6 \pm 1.4 \%\right.$ vs. $3.4 \pm 1.4 \%$ at $\mathrm{T}_{2}, 3.2 \pm 1.1 \%$ vs. $4.0 \pm 1.1 \%$ at $\mathrm{T}_{3}$ and $4.2 \pm 1.1 \%$ vs. $4.7 \pm 1 . \%$ at $\mathrm{T}_{4}$; all $\mathrm{P}<0.05$; Fig. 5). These results suggested that dexmedetomidine may decrease the percentages of MDSCs and improve the immunosuppressive state of patients.

Comparison of the incidence of emergence agitation, hypotension and bradycardia between the two groups. The incidence of emergence agitation was lower in the dexmedetomidine group compared with the control group (31.2 vs. $59.4 \%$; $\mathrm{P}=0.044)$. However, the incidence of hypotension and bradycardia during 
A
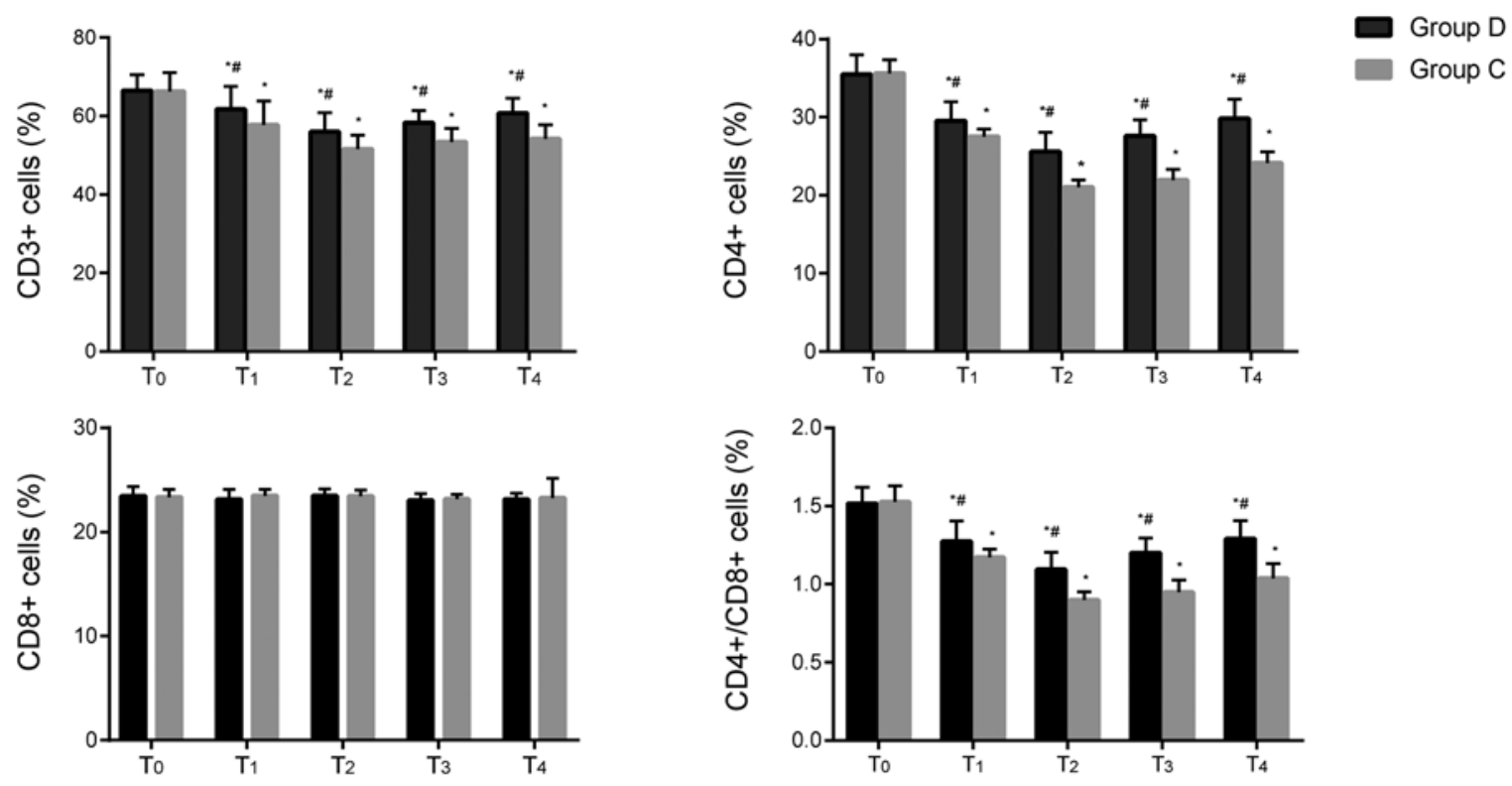

B
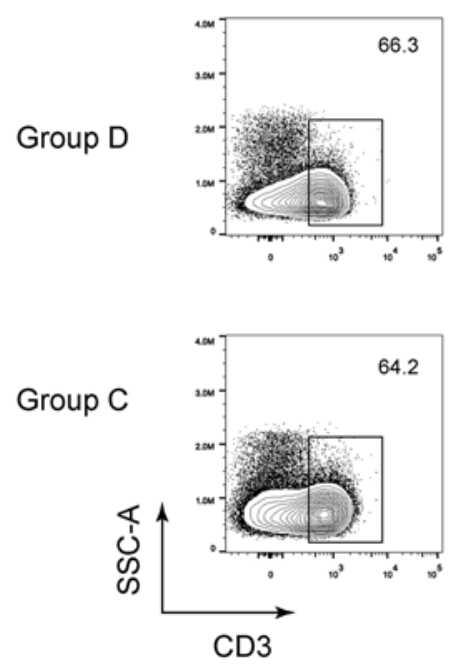

$\mathrm{T}_{1}$
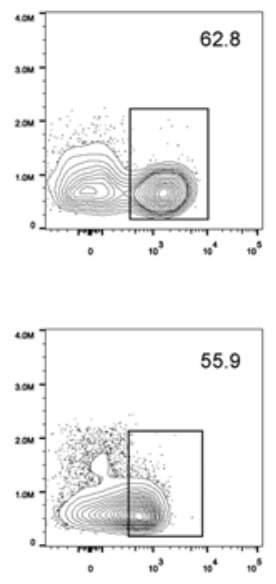

$T_{2}$
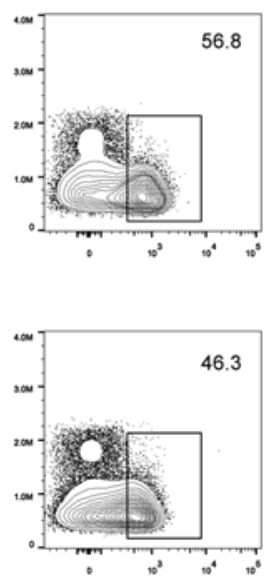

$\mathrm{T}_{3}$
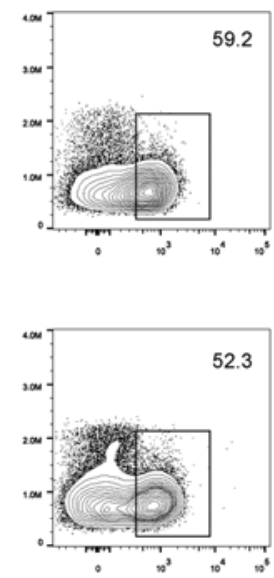

$\mathrm{T}_{4}$
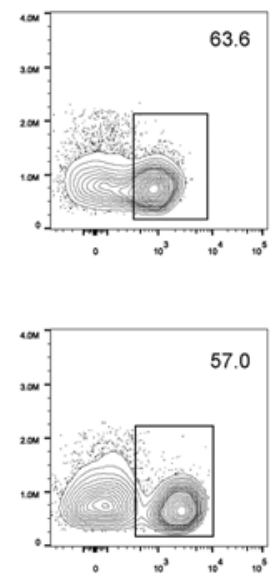

Figure 2. Comparison of T lymphocyte subsets at different time-points between the two groups. (A) Tlymphocyte subsets including $\mathrm{CD} 3^{+}, \mathrm{CD} 4^{+}$and $\mathrm{CD} 8^{+}$cells were analyzed by flow cytometry. Percentages of $\mathrm{CD}^{+}$and $\mathrm{CD} 4^{+}$cells, and the $\mathrm{CD} 4^{+} / \mathrm{CD}^{+}$ratios significantly decreased at $\mathrm{T}_{1-4}$ in the two groups compared with the baseline value at $\mathrm{T}_{0}$ and significantly increased at $\mathrm{T}_{1-4}$ in the dexmedetomidine group compared with the control group. ${ }^{*} \mathrm{P}<0.05$ vs. T0, ${ }^{\prime \prime} \mathrm{P}<0.05$ vs. group C. (B) Representative flow cytometry contour plots of $\mathrm{CD}^{+} \mathrm{T}$ cells in CD45+ cells of group C and group D at $\mathrm{T}_{0-4}$. $\mathrm{D}$, dexmedetomidine; $\mathrm{C}$, control; $\mathrm{T}_{0}$, $30 \mathrm{~min}$ before induction; $\mathrm{T}_{1}, 1 \mathrm{~h}$ after induction; $\mathrm{T}_{2}$, end of the operation; $\mathrm{T}_{3}, 24 \mathrm{~h}$ after operation; $\mathrm{T}_{4}, 48 \mathrm{~h}$ after operation.

surgery were significantly higher in the dexmedetomidine group compared with in the control group $(\mathrm{P}<0.05)$ (Table II).

\section{Discussion}

The present results showed that dexmedetomidine alleviated the decrease in the percentages of $\mathrm{CD}^{+}, \mathrm{CD}^{+}$and dendritic cells as well as the $\mathrm{CD} 4^{+} / \mathrm{CD} 8^{+}$ratios and reduced the percentages of MDSCs, indicating that dexmedetomidine can attenuate immunosuppression in patients undergoing radical and reconstructive surgery for oral cancer.

Oral cancer is a major life-threatening disease with a high incidence in Southeast Asian countries (1), and radical operation and immediate reconstruction are the common treatment for patients. The surgical stress response is considered to directly induce immunosuppression by activating the hypothalamus-pituitary-adrenal axis and sympathetic nervous system, which results in the increased production of glucocorticoids and catecholamines (3). Glucocorticoids are known to decrease the number and activity of natural killer cells and reduce $\mathrm{T}$ cell proliferation in a dose-dependent manner (17). Catecholamines can also inhibit $\mathrm{T}$ cell proliferation by decreasing IL-2 expression and secretion and reduce natural killer cell activity (3). Meanwhile, it is reported that some anesthetics have direct suppressive impacts on innate and adaptive immunity (5-8). Sevoflurane is a popular inhaled 
A

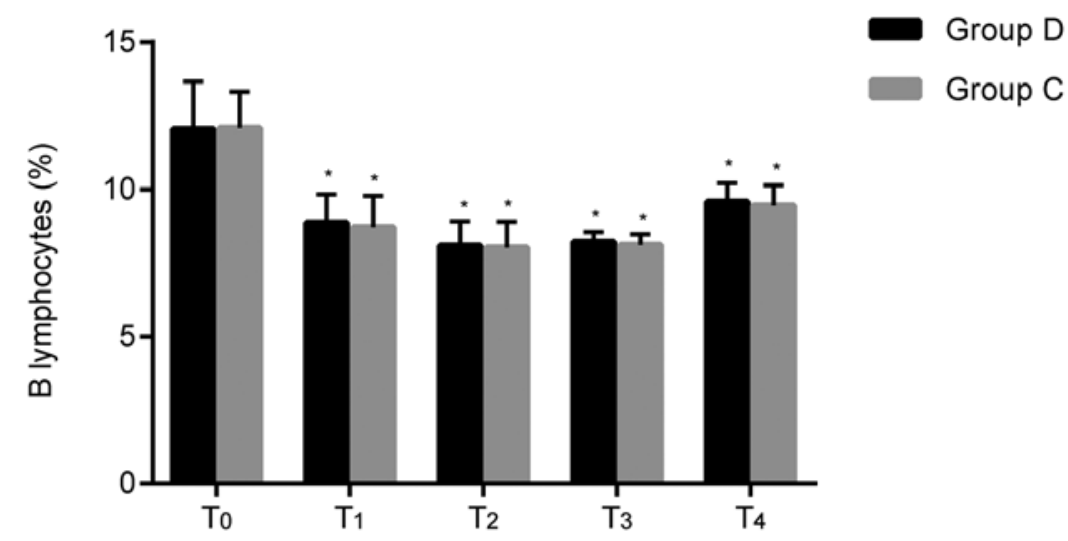

B

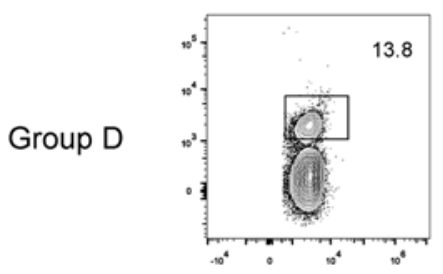

To

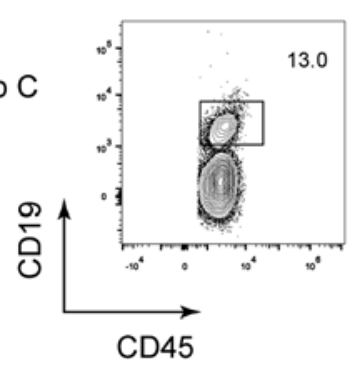

$T_{1}$
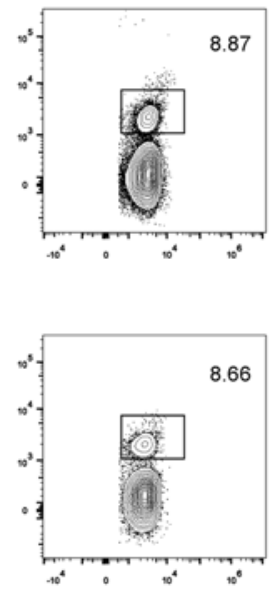

$\mathrm{T}_{2}$
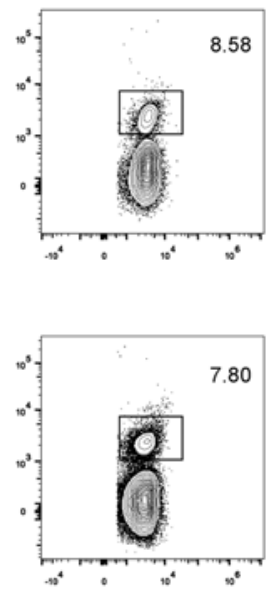

$\mathrm{T}_{3}$
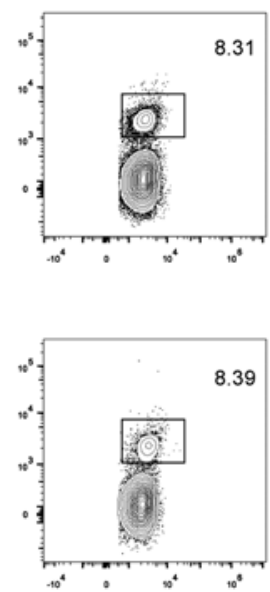

$\mathrm{T}_{4}$
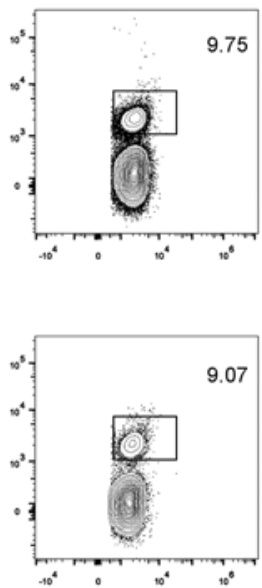

Figure 3. Comparison of B lymphocytes at different time-points between the two groups. (A) B lymphocytes were analyzed using flow cytometry. No significant differences were found between the two groups at the same time-points. ${ }^{*} \mathrm{P}<0.05$ vs. $\mathrm{T}_{0}$. (B) Representative flow cytometry contour plots of $\mathrm{B}$ lymphocytes in $\mathrm{CD}_{4} 5^{+}$cells of group $\mathrm{C}$ and group D. D, dexmedetomidine; $\mathrm{C}$, control; $\mathrm{T}_{0}, 30 \mathrm{~min}$ before induction; $\mathrm{T}_{1}, 1 \mathrm{~h}$ after induction; $\mathrm{T}_{2}$, end of the operation; $\mathrm{T}_{3}, 24 \mathrm{~h}$ after operation; $\mathrm{T}_{4}, 48 \mathrm{~h}$ after operation.

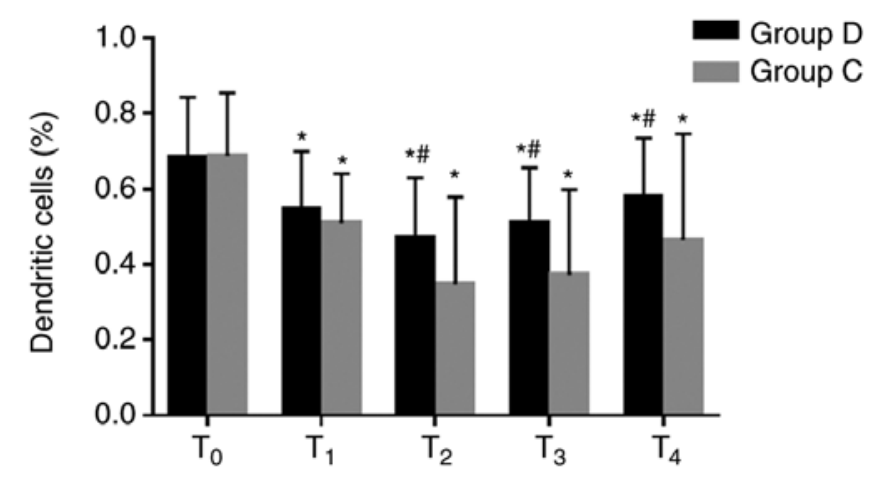

Figure 4. Comparison of dendritic cells at different time-points between the two groups. Percentages of dendritic cells were significantly higher at $\mathrm{T}_{2-4}$ in the group $\mathrm{D}$ compared with the group $\mathrm{C}$. ${ }^{*} \mathrm{P}<0.05$ vs. $\mathrm{T}_{0},{ }^{*} \mathrm{P}<0.05$ vs. group $\mathrm{C}$ $\mathrm{D}$, dexmedetomidine; $\mathrm{C}$, control; $\mathrm{T}_{0}, 30$ min before induction; $\mathrm{T}_{1}, 1 \mathrm{~h}$ after induction; $\mathrm{T}_{2}$, end of the operation; $\mathrm{T}_{3}, 24 \mathrm{~h}$ after operation; $\mathrm{T}_{4}, 48 \mathrm{~h}$ after operation.

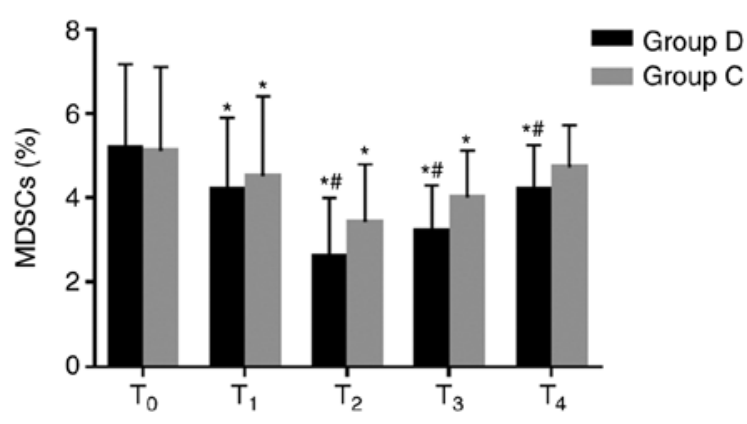

Figure 5. Comparison of MDSCs at different time-points between the two groups. MDSCs were analyzed using flow cytometry. Percentages of MDSCs significantly decreased at T1-3 in the two groups compared with the baseline value at $\mathrm{T} 0$, and they were significantly lower at T2-4 in the dexmedetomidine group compared with the control group. ${ }^{*} \mathrm{P}<0.05$ vs. $\mathrm{T} 0,{ }^{\#} \mathrm{P}<0.05$ vs. group C. MDSCs, myeloid-derived suppressor cells; D, dexmedetomidine; $\mathrm{C}$, control; T0, 30 min before induction; T1, $1 \mathrm{~h}$ after induction; T2, end of the operation; T3, $24 \mathrm{~h}$ after operation; T4, $48 \mathrm{~h}$ after operation. 
Table II. Incidence of emergence agitation, hypotension and bradycardia between the D $(n=32)$ and C $(n=32)$ groups.

\begin{tabular}{lccr}
\hline Variable & Group D, n (\%) & Group C, n (\%) & P-value \\
\hline Emergence agitation & $10(31.2)$ & $19(59.4)$ & 0.044 \\
Hypotension in OR & $14(43.8)$ & $5(15.6)$ & 0.027 \\
Bradycardia in OR & $12(37.5)$ & $3(9.4)$ & 0.016 \\
\hline
\end{tabular}

$\mathrm{D}$, dexmedetomidine, $\mathrm{C}$, control; OR, operating room.

anesthetic that can inhibit the activity of natural killer cells and induce the apoptosis of $\mathrm{T}$ and $\mathrm{B}$ lymphocytes (8). Opioids are commonly used analgesic agents in surgery and have immunosuppressive effects by suppressing the activity of natural killer cells, neutrophils and macrophages and the proliferation of T lymphocytes (7). Surgery and anesthesia-induced immunosuppression have been implicated in the development of post-operative septic complications and tumor metastasis $(3,4)$.

Considering that surgery is necessary in cancer treatment, extensive research has been conducted to determine the effect of anesthetics on immune cell population (5-8). Dexmedetomidine is a highly selective $\alpha_{2}$-adrenoceptor agonist with sedative, anxiolytic, analgesic and anti-sympatholytic properties $(9,10)$. Ebert et al $(18)$ reported that dexmedetomidine reduces the concentration of circulating plasma catecholamines by $60-80 \%$ in a dose-dependent manner, and this reduction is consistent with long-lasting anti-sympathetic effect. Other studies have indicated that dexmedetomidine exerts anti-inflammatory effects and organ-protective effects against ischemic and hypoxic injury, which can also regulate immune response $(10,13,19)$.

Cellular immunity is closely associated with antitumor effects and cancer metastasis surveillance $(6,20)$. Improving perioperative cellular immune status contributes to attenuation of immunosuppression and resistance to metastasis. T lymphocytes are important in cellular immunity (20). All mature peripheral $\mathrm{T}$ lymphocytes, labeled by $\mathrm{CD}^{+}$, comprise of $\mathrm{CD}^{+}$and $\mathrm{CD}^{+}$. The former represents cellular immune function, whereas the latter recognizes and kills tumor cells. The reduction of $\mathrm{CD}^{+} / \mathrm{CD}^{+}$ratio implies that the cellular immune function is downregulated (6). The present study showed that the percentages of $\mathrm{CD}^{+}$and $\mathrm{CD}^{+}$cells and the $\mathrm{CD}^{+} / \mathrm{CD}^{+}$ratios significantly decreased from $\mathrm{T}_{1}$ to $\mathrm{T}_{4}$ in the two groups and significantly decreased in the control group. This result implied that cellular immunity was suppressed in the two groups after anesthesia and dexmedetomidine may be associated with less impairment of cellular immunity in these patients. These results are aligned with previous reports. For example, Yang et al (11) found a significant difference in lymphocyte count after anesthesia between the dexmedetomidine and control groups in radical mastectomy. A recent clinical trial on 141 patients receiving radical operation of colon carcinoma has revealed that dexmedetomidine can decrease the inhibition of $\mathrm{T}$ lymphocyte subsets and reduce the secretion of inflammatory factors (12). Another study by Wang et al (13) indicated that dexmedetomidine can preserve the balance of $\mathrm{T}$ helper (h)1/Th2 ratio $24 \mathrm{~h}$ after surgery and attributed this result to the increased response of Th1 in patients undergoing radical gastrectomy. The decrease in $\mathrm{Th} 1 / \mathrm{Th} 2$ ratio after surgery suggests a suppressed cell-mediated immunity (13).

B lymphocytes producing antibodies mediate humoral immunity (6). Surgery-induced immunosuppression is mainly caused by the effect on the cellular immune system, and T lymphocytes are most affected with B lymphocytes numbers changing little (3). In the present study, the percentage of B lymphocytes was slightly decreased in the two groups after anesthesia, and there was no significant difference between the two groups at $\mathrm{T}_{1-4}$. The results suggested that dexmedetomidine exerts minimal effect on the humoral immune response of patients undergoing radical and reconstructive surgery for oral cancer. Further studies are needed to find the influence of dexmedetomidine on the humoral immunity of patients with malignant tumors during operation.

Dendritic cells, first identified by Steinman in 1973, are antigen-presenting cells that are considered a critical factor in anti-tumor immunity (21). As an immune surveillance cell, dendritic cells can efficiently cluster and activate $\mathrm{T}$ cells inhibiting the occurrence and development of tumors (22). Clinical studies on dendritic cells have focused on therapeutic vaccination against cancer, immunotherapy applying ex vivo-generated and tumor antigen-loaded dendritic cells has been successfully introduced in clinical vaccination protocols and has proven to be feasible and effective (23). The present study demonstrated that the percentages of dendritic cells were significantly higher in the dexmedetomidine group compared within the control group from $\mathrm{T}_{2}$ to $\mathrm{T}_{4}$. This result suggested that dexmedetomidine may attenuate the inhibition of immune response and may be beneficial to antitumor therapy.

MDSCs are a subset of immune cells that have a myeloid origin with immunosuppressive abilities (24). Studies have reported that MDSCs suppress $\mathrm{CD}^{+} \mathrm{T}$ cells and IFN- $\gamma$ and IL-2 production by $\mathrm{T}$ cells $(24,25)$. In addition, MDSCs inhibit B cell proliferation and antibody production (25) and the expansion of MDSCs is associated with tumor progression (26). The present study showed that the percentages of MDSCs significantly decreased from $T_{1}$ to $T_{3}$ in the two groups, indicating that surgical resection of the tumor has an anti-tumor effect. Meanwhile, the percentages of MDSCs in the dexmedetomidine group significantly decreased at $T_{2}$ to $T_{4}$, suggesting that dexmedetomidine may decrease the percentages of MDSCs and improve the immunosuppressive state of patients.

The level of agitation during emergence was assessed using RASS scores (16). The incidence of emergence agitation was lower in the dexmedetomidine group compared 
within the control group. This result may be associated with the sedation and analgesia effects of dexmedetomidine. Thus, patients are likely to tolerate tracheal catheter and other discomfort during the recovery period. However, the incidence of hypotension and bradycardia was higher in the dexmedetomidine group compared within the control. This result is in accordance with previous research and the reason is related to the inhibitory effect of dexmedetomidine on the sympathetic system $(27,28)$.

The present study has some limitations. The clinical outcomes and cancer-free survival time were not measured. In addition, the specific mechanisms of immunosuppressive effects of dexmedetomidine were not explored. Therefore, cellular and molecular level studies are also needed to resolve these underlying mechanisms. Immune cells could be analyzed to detect mRNA expression levels of transcription factors and chemokines.

In conclusion, dexmedetomidine alleviated the decrease in the percentages of $\mathrm{CD}^{+}, \mathrm{CD}^{+}$and dendritic cells as well as the $\mathrm{CD} 4^{+} / \mathrm{CD}^{+}$ratios and reduced the percentages of MDSCs. Thus, dexmedetomidine can attenuate immunosuppression in patients undergoing radical and reconstructive surgery for oral cancer.

\section{Acknowledgements}

The authors would like to thank their colleague Dr Liang Mao (School and Hospital of Stomatology, Wuhan University, Wuhan, Hubei, China) for his excellent technical assistance.

\section{Funding}

No funding was received.

\section{Availability of data and materials}

All data generated or analyzed during this study are included in this published article.

\section{Authors' contributions}

LH designed the study, performed the experiments and wrote the manuscript. CQ performed the experiments and revised the manuscript. LW designed the study and revised the manuscript. TZ collected and analyzed the data. JL designed the study and revised the manuscript. All authors read and approved the final manuscript.

\section{Ethics approval and consent to participate}

This study was approved by The Ethics Committee of School and Hospital of Stomatology, Wuhan University (Wuhan, China; approval noIRB-2018B23) and registered in the Chinese Clinical Trial Registry (ChiCTR-1800018367). Written informed consent was provided by all participants before the trial.

\section{Patient consent for publication}

Not applicable.

\section{Competing interests}

The authors declare that they have no competing interests.

\section{References}

1. Kumar M, Nanavati R, Modi TG and Dobariya C: Oral cancer: Etiology and risk factors: A review. J Cancer Res Ther 12: 458-63, 2016

2. Hussein AA, Helder MN, de Visscher JG, Leemans CR, Braakhuis BJ, de Vet HCW and Forouzanfar T: Global incidence of oral and oropharynx cancer in patients younger than 45 years versus older patients: A systematic review. Eur J Cancer 82: 115-127, 2017.

3. Hogan BV, Peter MB, Shenoy HG, Horgan K and Hughes TA: Surgery induced immunosuppression. Surgeon 9: 38-43, 2011.

4. Kim R: Anesthetic technique and cancer recurrence in oncologic surgery: Unraveling the puzzle. Cancer Metastasis Rev 36: 159-177, 2017.

5. Kurosawa $\mathrm{S}$ and Kato $\mathrm{M}$ : Anesthetics, immune cells, and immune responses. J Anesth 22: 263-277, 2008.

6. Zhang T, Fan Y, Liu K and Wang Y: Effects of different general anaesthetic techniques on immune responses in patients undergoing surgery for tongue cancer. Anaesth Intensive Care 42: 220-227, 2014.

7. Plein LM and Rittner HL: Opioids and immune system-friend or foe. Br J Pharmacol 175: 2717-2725, 2018.

8. Stollings LM, Jia LJ, Tang P, Dou H, Lu B and Xu Y: Immune modulation by volatile anesthetics. Anesthesiology 125: 399-411, 2016.

9. Mahmoud M and Mason KP: Dexmedetomidine: Review, update, and future considerations of pediatric perioperative and periprocedural applications and limitations. Br J Anaesth 115: 171-182, 2015.

10. Maud AS, Michel MR, Laura NH, Clements RM, Anthony RA and Pieter C: Clinical pharmacokinetics and pharmacodynamics of dexmedetomine. Clin Pharmacokinet 56: 893-913, 2017.

11. Yang XH, Bai Q, Lv MM, Fu HG, Dong TL and Zhou Z: Effect of dexmedetomidine on immune function of patients undergoing radical mastectomy. Eur Rev Med Pharmacol Sci 21: 1112-1116, 2017.

12. Wang $\mathrm{K}$ and Li C: Effects of dexmedetomidine on inflammatory factors, $\mathrm{T}$ lymphocyte subsets and expression of NF- $\kappa \mathrm{B}$ in peripheral blood mononuclear cells in patients receiving radical surgery of colon carcinoma. Oncol Lett 15: 7153-7157, 2018.

13. Wang Y, Xu X, Liu H and Ji F: Effects of dexmedetomine on patients undergoing radical gastrectomy. J Surg Res 194: 147-153, 2015.

14. Mayhew D, Mendonca V and Murthy BVS: A review of ASA physical status-historical perspectives and modern developments. Anesthesia 74: 373-379, 2019.

15. Scott NW, McPherson GC, Ramsay CR and Campbell MK: The method of minimization for allocation to clinical trials: A review. Control Clin Trials 23: 662-674, 2002.

16. Barr J, Fraser GL, Puntillo K, Ely EW, Gélinas C, Dasta JF, Davidson JE, Devlin JW, Kress JP, Joffe AM, et al: Clinical practice guidelines for the management of pain, agitation, and delirium in adult patients in the Intensive Care Unit. Crit Care Med 41: 263-306, 2013.

17. Chen L, Jondal $M$ and Yakimchuk $K$ : Regulatory effects of dexamethasone on NK and T cell immunity. Inflammopharmacology 26: 1331-1338, 2018.

18. Ebert TJ, Hall JE, Barnev JA, Uhrich TD and Colinco MD: The effects of increasing plasma concentrations of dexmedetomidine in humans. Anesthesiology 93: 382-394, 2000.

19. Xiang H, Hu B, Li Z and Li J: Dexmedetomine controls systemic cytokine levels through the cholinergic anti-inflammatory pathway. Inflammation 37: 1763-1770, 2014.

20. Goldfarb Y, Sorski L, Benish M, Levi B, Melamed R and Ben-Eliyahu S: Improving postoperative immune status and resistance to cancer metastasis: A combined perioperative approach of immunostimulation and prevention of excessive surgical stress responses. Ann Surg 253: 798-810, 2011.

21. Steinman RM: Decisions about dendritic cells: Past, present, and future. Annu Rev Immunol 30: 1-22, 2012.

22. Mildner A and Jung S: Development and function of dendritic cell subsets. Immunity 40: 642-656, 2014. 
23. Den Brok MH, Nierkens S, Figdor CG, Ruers TJ and Adema GJ: Dendritic cells: Tools and targets for antitumor vaccination. Expert Rev Vaccines 4: 699-710, 2005.

24. Dai J, El Gazzar M, Li GY, Moorman JP and Yao ZQ: Myeloid-derived suppressor cells: Paradoxical roles in infection and immunity. J Innate Immun 7: 116-126, 2015.

25. Tamadaho RSE, Hoerauf A and Layland LE: Immunomodulatory effects of myeloid-derived suppressor cells in diseases: Role in cancer and infections. Immunobiology 223: 432-442, 2018.

26. Marvel D and Gabrilovich DI: Myeloid-derived suppressor cells in the tumor microenvironment: Expect the unexpected. J Clin Invest 125: 3356-3364, 2015.

27. Yang X, Li Z, Gao C and Liu R: Effect of dexmedetomidine on preventing agitation and delirium after microvascular free flap surgery: A randomized, double-blind, control study. J Oral Maxillofac Surg 73: 1065-1072, 2015.
28. Riker RR, Shehabi Y, Bokesch PM, Ceraso D, Wisemandle W, Koura F, Whitten P, Margolis BD, Byrne DW, Ely EW, et al: Dexmedetomidine vs. midazolam for sedation of critically ill patients: A randomized trial. JAMA 301: 489-499, 2009.

(c) (i) () $९$ This work is licensed under a Creative Commons c) Attribution-NonCommercial-NoDerivatives 4.0 International (CC BY-NC-ND 4.0) License. 